This is an Accepted Manuscript of

G. Mazzanti et al., "The insulation of HVDC extruded cable system joints. Part 2: Proposal of a new AC voltage PD measurement protocol for quality control during routine tests,"

in:

IEEE Transactions on Dielectrics and Electrical Insulation, vol. 26, no. 3, pp. 973980, June 2019

The final published version is available online at:

https://doi.org/10.1109/TDEI.2019.007917

Rights / License:

The terms and conditions for the reuse of this version of the manuscript are specified in the publishing policy. For all terms of use and more information see the publisher's website.

This item was downloaded from IRIS Università di Bologna (https://cris.unibo.it/)

When citing, please refer to the published version. 


\title{
The Insulation of HVDC Extruded Cable System Joints. Part 2: Proposal of a New AC Voltage PD Measurement Protocol for Quality Control during Routine Tests
}

\author{
Prepared by the IEEE DEIS HVDC Cable Systems Technical Committee
}

\section{G. Mazzanti, Chair}

Dept. of El. Energy Engin. Information

University of Bologna

Viale Risorgimento 2

Bologna 40136, Italy

\section{J.C. Fothergill}

Pro Vice-Chancellor, City University

London, Northampton Square

London EC1V 0HB, UK

\section{J.H. Lee}

Dept. of Electrical Engineering

Hoseo University

Asan, Chungcheongnam-do

Asan 336-010, South Korea

\section{F. Mauseth}

Norw. Univ. of Science and Tech.

Dept.of Electric Power Engineering

O.S. Bragstads Plass 2E

Trondheim 7491, Norway

\section{Troia}

Prysmian Cables \& Systems

Research \& Development Headquarters

Via Ansaldo

Milan 20100, Italy
J.Castellon

Institute of Electronics and Systems -

University of Montpellier

860 rue de Saint Priest

Montpellier 34090, France

\section{Fu}

Electric Power Research Institute

China Southern Power Grid

Guangzhou 510080, China

\section{J. Li}

Dept. High Voltage \& Insulation Eng.

College of Electrical Eng.

Chongqing University

Chongqing 400044, China

\section{P. Morshuis}

Solid Dielectric Solutions

Herensteeg 17, 2311 SG Leiden

The Netherlands

\section{A. Tzimas}

Advanced Energy

High voltage products group

Unit 10, Hawthorn Road,

Littlehampton,W.Sussex, BN177LT,UK
G. Chen

Electronics and Computer Sciences University of Southampton University Road, Highfield Southampton SO17 1BJ, UK

\section{N. Hozumi}

Dept. El. \& Electronic Inform. Engin. Toyohashi University of Technology

Toyohashi 441-8580, Japan

\section{Marzinotto}

TERNA

Engineering Department Via della Marcigliana 911

Roma 00138, Italy

\author{
C. Reed \\ USA Consultant \\ Private Consultant \\ Scotia, New York 12302 \\ USA
}

\section{K. Wu}

State Key Lab. of Electr. Insul. Power Equip., Xi' an Jiaotong University

28 Xianning West Road Xi'an 710049, China

\begin{abstract}
The review of materials, design and testing of joints for HVDC extruded cable systems provided in previous Part 1 paved the way to this Part 2 position paper by the DEIS HVDC Cable Systems Technical Committee, whose aim is to remedy the scarcity of existing standardized tests on joints. After a sound analysis, here routine tests are identified as the first practical target for the onset of new testing procedures, AC-PD measurements as the readily-available measurement from manufacturers' experience for quality control of joints during routine tests and VHF/UHF wireless sensors as the best tool for such measurements in the noisy environment of factories. Thereby, a novel protocol for PD measurement using AC voltages and VHF/UHF electromagnetic sensors, for quality control during routine tests on HVDC extruded joints, is proposed.

Index Terms - HVDC insulation, joints, partial discharges, power cable systems, power cable testing, wireless sensors, VHF/UHF electromagnetic sensors
\end{abstract}




\section{INTRODUCTION}

AS emphasized in the previous Part 1 of this paper [1], High Voltage Direct Current (HVDC) cable systems with extruded insulation are becoming more and more attractive vs. Mass Impregnated Non-Draining (MIND) HVDC cable systems [2], but still have weak points.

One is space charge accumulation under the applied DC voltage. The IEEE DEIS Technical Committee (TC) on "HVDC Cable Systems (cables, joints and terminations)" has addressed this issue yielding IEEE Std. 1732-2017 entitled "Recommended practice for space charge measurements in HVDC extruded cables for rated voltages up to $550 \mathrm{kV}$ ' [3, 4].

Another issue is the development of long-lasting and reliable accessories, namely joints and terminations. Here, following the suggestions by CIGRÉ Technical Brochure (TB) 496 [5] and IEC Standard 62895 [6] about developing new tests for HVDC cable system accessories the TC has decided that its next main task should be identification of techniques to characterize accessories, in particular joints, for qualification and routine tests. In the previous Part 1, the TC - after verifying the lack of feasibility to date of using space charge measurement techniques in qualification and routine tests for joints - has focused on PD measurements, as suggested also in $[5,6]$.

This paper shows that (Section 2):

1) routine tests are the first practical target for the onset of new PD measurements on joint insulation;

2) $\mathrm{PD}$ measurements under $\mathrm{AC}$ voltage are the readilyavailable measurement from manufacturers' practices for quality control of the insulation of accessories during routine tests;

3) VHF/UHF electromagnetic (wireless) sensors are the best tool for performing such measurements on joints effectively in noisy environments like factories.

Then, in Section 3, the basics of VHF/UHF electromagnetic sensors are recalled and in Section 4, a new PD-based protocol for quality control during routine tests for HVDC extruded cable system joints is proposed.

\section{PD MEASUREMENTS ON THE INSULATION OF JOINTS: WHEN AND HOW?}

In fact, so far PD measurements on HVAC cable systems have been selected as a reference for both on-site testing $[7,8]$ and routine tests in the factory $[9,10]$, these latter to be carried out in accordance with IEC 60885-3 [11]. For HVDC cables, as seen above, both CIGRE TB 496 and IEC 62895 suggest $\mathrm{AC}$ voltage tests combined with $\mathrm{PD}$ measurements as factory tests for cable joints when possible; however, the test procedures (type of power supply, voltage level, etc.) are left to the discretion of customer and manufacturer. This selection is not trivial, as the conventional PD measurement using Frequency-tuned Resonant AC (ACRF) supply on longdistance HVDC power cable systems with large capacitance first of all challenge the voltage rating of power source [12]. In most cases, commercially available Damped AC (DAC) testing equipment have rated voltage $\leq 400 \mathrm{kVpeak}$ (283 $k V r m s$ ) and Very Low Frequency (VLF) rated voltage $\leq 200$ kVpeak (140 kVrms) [7]. Furthermore it is hard to reach an agreement between customer and manufacturer on type of power supply, voltage level and duration of the AC test, also because such a test involves space charge-related problems if carried out after HVDC testing [7].

Alternatively, one might consider DC PD testing. In this respect, as pointed out in [7] "researchers of TU Delft established a fundamental framework for PD detection and analysis under DC voltage, suggesting the time interval $\Delta t$ between adjacent discharges as the substitute for the phase information under $A C$, along with charge magnitude $q$ to describe PD activities under DC... [13]. However, a basic consensus of how to conduct DC PD measurement for cable systems is still absent and PD detection conducted on actual HVDC extruded cable loops has not been reported yet" [7]. One of the few possible references for setting procedures and limits for DC-PD measurements on HVDC cable system accessories is IEC/IEEE 65700-19-03:2014 relevant to "Bushings for DC application" [14]. Of course, its application to HVDC cable system accessories is more than questionable. In addition, a correlation has not been found yet between the PDs detected under DC voltage and the real aging (and failure) of the components of a HVDC cable system ${ }^{1}$.

As there is not enough confidence at present about the test procedures, the determination of the quantities, the levels of voltage to be applied and the limits to be prescribed for DC PD measurements in cable systems, PD measurements at the qualification/routine stage should necessarily involve PD measurements under $\mathrm{AC}$ voltage, i.e. AC-PD measurements.

A first proposal within the TC was AC-PD measurements on the insulation of joints during qualification tests of HVDC cable systems. Unfortunately, PD measurements under AC voltage at the qualification stage are difficult to be performed in practice, as they would necessarily require the presence of a HVAC voltage generator at the same time as the HVDC voltage generator and impulse voltage generator already required for prequalification and type tests. This makes managing the space in the lab and the whole sequence of qualification tests quite cumbersome, costly and timeconsuming, at least with the available technology of AC, DC and impulse test generators; anyway, this situation is not expected to change by much in the near future.

Furthermore, it must be emphasized that the meaning of AC-PD measurements at the qualification stage would only be checking the correctness of the installation of the joint in the qualification cable loop, as manufacturers' experience proves

\footnotetext{
1 In this respect, IEC 60270 at $\$ 11.5 .1$ highlights that "The procedures described for alternating voltage to determine the PD inception and extinction voltages are generally not applicable for tests with direct voltage as the stress on the dielectric during voltage rise and decrease is different from that during the period when the voltage is constant. There is no accepted general method for the determination of partial discharge quantities during tests with direct voltage. Whatever method is used, it is important to note that magnitudes related to partial discharges at the beginning of the voltage application can be different from the magnitudes measured after a considerable time at the same test". CIGRE WG D1.63 deals with PDs under HVDC.
} 
that PDs detected in the joint of a qualification loop are associated with errors in mounting the joint rather than with inner defects in the joint insulation. On the contrary, the meaning of AC-PD measurements at the routine test stage is checking the quality of the insulation of a certain joint design, realized with a certain manufacturing procedure. These two checks are of course useful and complementary to each other, but as a matter of fact AC-PD measurements are too invasive on the time schedule and the costs of qualification tests.

For this reason, as a first, feasible step in the direction of developing Standards relevant to accessories, the TC on "HVDC Cable Systems" has decided to focus on AC voltage routine test with PD measurements on factory and prefabricated joints. This is not only in line with the suggestions after TB 496 and IEC 62895 (see Section 3.1) but also in agreement with quality control practices already implemented by major cable manufacturers worldwide, which already perform AC-PD measurements on HVDC cable system joints as routine tests in the factory, in particular on:

- Factory Flexible Joints (FFJ) for submarine cables;

- Pre-moulded Joints for land cables.

Moreover, according to main manufacturers' experience, such measurements cannot be done using traditional PD measurement systems compliant with IEC 60270 [15], in particular for FFJ for submarine cables, for many reasons:

i) the huge delivery lengths of submarine cables makes calibration (to be done at terminations) very difficult;

ii) signal attenuation is dramatic on such huge delivery lengths;

iii) locating signals from the various FFJ is practically unfeasible;

iv) the factory environment is very noisy (this holds also for routine testing of pre-moulded joints in the factory).

Conversely, such measurements are done much more effectively and in a much easier way with PD VHF and/or UHF electromagnetic (EM) sensors. Unfortunately, PD VHF/UHF EM sensors miss a reference Standard, since IEC 60270 focuses on PD measurement devices that require a capacitive or inductive coupling device (CD).

Based on the above reasoning, the TC "HVDC Cable Systems" has decided to focus all its efforts on AC-PD measurements with VHF/UHF EM sensors during routine tests on HVDC cable system FFJ and pre-moulded joints. In this way, a twofold goal is achieved:

1) the selected goal of introducing new electrical characterization techniques for testing HVDC cable system accessories, in particular joints;

2) the additional goal of taking a first step towards the Standardization of VHF/UHF EM sensors.

Some considerations of the main features of VHF/UHF EM sensors are reported in the next Section, while a first proposal of a recommended protocol for AC-PD measurements with VHF/UHF EM sensors during routine tests on HVDC cable system FFJ and pre-moulded joints is reported in Section 5.

\section{PD MEASUREMENTS USING VHF/UHF ELECTROMAGNETIC SENSORS}

The operation principle of VHF/UHF EM PD sensors is comparable with that of antennas operating in the near-field region. That means the output signal is determined through both the electric field vector, $\vec{E}$, and the magnetic field vector, $\vec{H}$, as given by the well-known coupled Maxwell equations [16]:

$$
\begin{gathered}
\nabla \times \vec{H}=\vec{J}+\varepsilon \cdot \frac{\partial \vec{E}}{\partial t} \\
\nabla \times \vec{E}=-\mu \cdot \frac{\partial \vec{H}}{\partial t}
\end{gathered}
$$

where $\vec{J}$ is current density, $\varepsilon$ is dielectric permittivity and $\mu$ is magnetic permeability of the medium where the propagation of the output signal takes place.

Devices of this kind have been - and are being - developed worldwide, starting in particular from UHF electro-magnetic sensors for PD measurements in GIS [17]. Depending on the geometrical configuration of the test object, various kinds of EM sensors are used for PD detection in the VHF/UHF range, such as rod, disc, conical, etc. antennas [16]. When integrated with a high performance acquisition system and a wireless (WiFi) interface, the sensor is capable to perform on-line PD measurements on the insulation of powered components at a proper distance from the tested object, without any galvanic connection $[18,19]$.

As an example, the instrument described in [19] falls in the class of Ultra-wide band (UWB) systems, as its wideband antenna has an almost flat gain and a linear phase dependence within the typical frequency band of PD signals in power cables $(0.1 \mathrm{MHz}-100 \mathrm{MHz})^{2}$. The antenna has a directional behavior, so during PD measurements the instrument has to be directed towards the test object [19]. PD pulses are acquired, stored, processed and sent to a computer by a Field Programmable Gate Array (FPGA) with a resolution of 8 bit and a rate of $200 \mathrm{MS} / \mathrm{s}$. The instrument, initially developed within a research activity carried out at the university of Palermo, Italy [20-22], includes a high-pass and a low-pass filter to obtain, respectively, PD-pulse signals and AC voltage phase reference. This way, it can locate the PD source accurately and provide in real time the Phase Resolved PD (PRPD) pattern for the identification of PD sources and the accurate shape of each pulse, so as to perform an effective separation of mixed PD patterns. Other wireless instruments for PD detection featuring VHF/UHF EM sensors are being

\footnotetext{
${ }^{2}$ A wideband antenna is one with approximately or exactly the same operating characteristics over a very wide passband. Ultra-wideband (UWB) is defined as an antenna transmission for which emitted signal bandwidth exceeds the lesser of $500 \mathrm{MHz}$ or $20 \%$ of the arithmetic center frequency.
} 
not only developed as laboratory instruments for research purposes, but also made commercially available worldwide (see e.g. [23-25]).

The main advantages of VHF/UHF EM sensors vs. IEC 60270-compliant instruments are that VHF/UHF EM sensors:

i) can reject the noise of factory environment, thanks to the neat increase of the signal-to-noise ratio, thereby enabling the attainment of clear phase-resolved partial discharge (PRPD) patterns. In fact, clear PRPD patterns are required by modern diagnostic procedures for the identification of defects generating PDs [16, 22];

ii) can detect the real shape of every PD pulse accurately in the time domain through their typically very wide band, thereby enabling the separation of PD pulses coming from different sources on the basis of their characteristic shapes. In fact, modern diagnostic procedures require accurate pulse-signal shapes to perform an effective separation of mixed PD patterns, since each different type of PD-generating defect (internal void, surface discharges, corona) has its typical pulse shape in the time domain $[16,21,22]$. A proper identification and separation of PD sources is essential to diagnose the condition of the joint;

iii) can be moved and located at the desired position of the joint, since they are wireless devices and do not need a direct coupling with the measured system. In this way signal attenuation along the cable is no more a problem and defect location at a joint is straightforward [16];

iv) can be calibrated via a proper wireless calibration procedure. This procedure is in fact similar to that of IEC 60270-compliant devices, as pointed out later in Section 5.1, and exploits the stray capacitance of the VHF/UHF EM sensor. Such calibration procedure is a novelty typical of last-generation VHF/UHF EM sensors $^{3}$ and is presently missing in Standards, but it could be normalized in a dedicated, innovative Recommended Practice (see Section 5).

All these advantages are particularly striking for AC-PD measurements in the factory on FFJ within the very long delivery lengths of submarine HVDC cable systems, but they are quite significant also for PD measurements in the factory on pre-moulded joints.

\section{PROPOSAL OF A NEW PD-BASED PROTOCOL FOR QUALITY CONTROL DURING ROUTINE TESTS}

The above review about the insulation of HVDC extruded cable system joints - dealing with existing materials, design and testing procedures - has emphasized, among the other issues concerning joint insulation, the following items:

\footnotetext{
${ }^{3}$ The literature on "traditional" UHF/VHF PD detection instruments typically claims that their signal cannot be calibrated in terms of $\mathrm{pC}$, thus the PD quantities measured by these instruments are not correlated with IEC 60270compliant instruments
}

- the lack for Standards and Recommended Practices about dedicated measurements and characterization tests on HVDC extruded cable system accessories;

- the potential usefulness of PD measurements in this respect;

- the great uncertainties with PD measurements under DC voltage in general;

- the difficulties with "conventional" - i.e. IEC 60270 compliant - PD measurements under AC voltage, in general and in particular during qualification tests;

- the great advantages of VHF/UHF EM sensors vs. IEC 60270-compliant instruments, especially for AC-PD measurements during routine tests in the factory on FFJ for submarine HVDC cable systems, as well as on premoulded joints for land cables;

- the lack of any Standardization about PD measurements with VHF/UHF EM sensors.

Considering these factors, in this Section - as a final contribution of this Position Paper - the IEEE DEIS TC on "HVDC Cable Systems (cables, joints and terminations)" proposes a new protocol for quality control during routine tests in the factory on FFJ for submarine HVDC extruded cable systems and on pre-moulded joints for land HVDC extruded cable systems. The protocol, to be further refined in a subsequent stage of this investigation by the TC, is based on PD measurements under AC voltage by means of VHF/UHF EM sensors and summarizes the good technical practices developed so far on these measurements during routine tests in the factory. The protocol establishes:

- the calibration (or sensitivity check) of the VHF/UHF EM sensor;

- the test setup;

- the measurement procedure;

- the acceptance criteria.

The main goal of this protocol is becoming an IEEE Recommended Practice for quality control to assess the manufacturing process and to exclude the presence of internal defects within the insulation of the joints that can lead to breakdown or accelerate ageing when the system will start the operation. In this respect, it should be noted that the results coming from such PD measurements is for the customer a very useful additional information that adds to the results of the simple AC voltage test. Such information may lead to reject a joint that would otherwise pass the AC voltage test, thereby avoiding subsequent failures in the next stages of the cable system life, i.e. after-installation test and service.

For this reason, an increasing number of customers is asking manufacturers this AC PD routine test on joints. Hence, a Recommended Practice on such test is really needed.

\subsection{SENSITIVITY CHECK (CALIBRATION)}

The protocol starts with the calibration of the PD sensor, which is similar to that of IEC 60270-compliant devices. The 
similarity points are as follows:

- a standard calibrator simulates the PD-generating defect exactly as for IEC60270 standard;

- known signal(s) are emitted by the calibrator;

- a coupling capacitance does in fact exist as for IEC60270 calibration: the capacitive coupling through the surrounding media.

The main difference lies in that, since the instrument is not physically connected to the circuit, the coupling is given by its position and orientation. On the other hand, as pointed out above, the absence of direct coupling is also one of the major advantages of the wireless instrument, which can be located at a short distance from the PD source (or from the calibrator during the calibration procedure), thus maximizing the sensitivity (signal to noise ratio). Another difference is that the calibration hereunder proposed is conceived for on-site measurements in the noisy environment of a factory, rather than for the lab, and is also called sensor "sensitivity check".

For a preliminary sensitivity check, a standard calibrator shall be connected to a wireless antenna, positioned at a distance from the sensor equal to the minimum distance achievable between the sensor and the measurement point: let us refer to this distance as "measurement distance" hereafter (see Figure 1). If the PD sensor is directional, the calibrator shall be positioned along the direction where the ratio signalto-noise of the PD sensor is maximum. Then, different trains of signals shall be subsequently emitted by the calibrator, at different progressively-decreasing amplitudes, and the sensitivity shall be identified as the minimum amplitude detectable by the PD instrument above the background noise ${ }^{4}$.

The sensitivity check can be done in the air near the sensor, or also on a sample cable in order to reproduce the propagation of the PD along the cable. A $1 \mathrm{~m}$ sample cable is enough to reproduce the propagation.

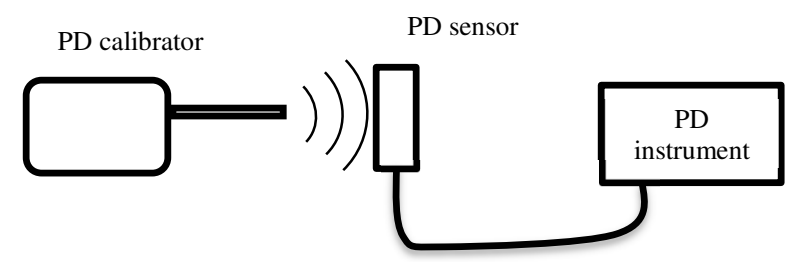

Figure 1. Sketch of the sensitivity check (calibration).

As far as possible specifications for the features of PD measurement instrument and of the calibrator, the following aspects should be highlighted.

- Experiments show that - independently of the source most frequency content of PDs is located in the VHF range and in particular from $1 \mathrm{MHz}$ to $100 \mathrm{MHz}$ and

\footnotetext{
${ }^{4}$ Of course such sensitivity is strongly dependent on the noise level of the environment and on the distance and direction of the sensor with respect to the signal source. This is the reason why the above prescriptions about distance and directions have been stated. It is also worth pointing out that PD antenna sensors usually display the acquired signal as a voltage signal in $\mathrm{mV}$.
}

above. The only PD's that have UHF content up to several hundred of $\mathrm{MHz}$ are related to GIS components, but however these PDs have also components in the VHF range, so they can be detected anyway using a VHF antenna. However, for a complete analysis of the PD phenomena, the instrument should be capable to acquire the PD pattern with amplitude, the wave shape in time domain and the frequency content in the range of the antenna sensor. Ideally, every pulse of the PD pattern should be acquired in a way that shape and frequency content can be discriminated. The amplitude can be recorded not only in $\mathrm{pC}$, but also in $\mathrm{dB}$ or in $\mathrm{mV}$, that is the output signal of the antenna sensor.

- Sensitivity will not be different for different PD fault types that may have different VHF/UHF frequencies if the instrument's antenna has a flat frequency response in the working range. As emphasized in Section 3, this is typically the case of a wideband antenna like those of VHF/UHF EM sensors for which the proposed calibration/ measurement procedure is conceived. In these case no matter the frequency of the calibrator pulse, the sensitivity of the sensor will be evaluated in the whole frequency spectrum. For this reason the antenna sensor should have a flat frequency response in order to acquire the different frequency components with the same gain. The shape of the sensor can be designed for this purpose, but in general directional antennas are preferred as they are less influenced from the surrounding irradiated EM noise.

- The calibrator can be a normal PD calibrator, it's just necessary that the pulse itself has frequency components above $1 \mathrm{MHz}$, but this condition is already satisfied.

\subsection{TEST SETUP}

The test setup shall consist of:

- the test object (either a FFJ or a pre-moulded joint) positioned within a cable system loop in the factory;

- the PD instrument and the relevant PD sensor;

- a suitable HVAC power source ${ }^{5}$ properly connected to the cable system loop.

The sensor shall be placed at the measurement point, which shall be selected within $10 \mathrm{~m}$ along the cable section including the test object because of the possible attenuation of the highest frequency components. Indeed, the frequency components above $50 \mathrm{MHz}$ will be attenuated within a few tens of meters while travelling along the cable (see Figure 3 ). Thus, keeping the measurement point within $10 \mathrm{~m}$ along the cable section including the joint ensures the best conditions to analyze a possible PD phenomenon while measuring.

It must be pointed out that for FFJ there is no need to connect the metal screen/sheath to the ground near the joint, as usually done with HFCT sensors (see Figure 2), with the great advantage of keeping the noise level low.

\footnotetext{
${ }^{5}$ In the case of FFJ the HVAC source will likely be a resonant test circuit operated at a proper frequency [7].
} 


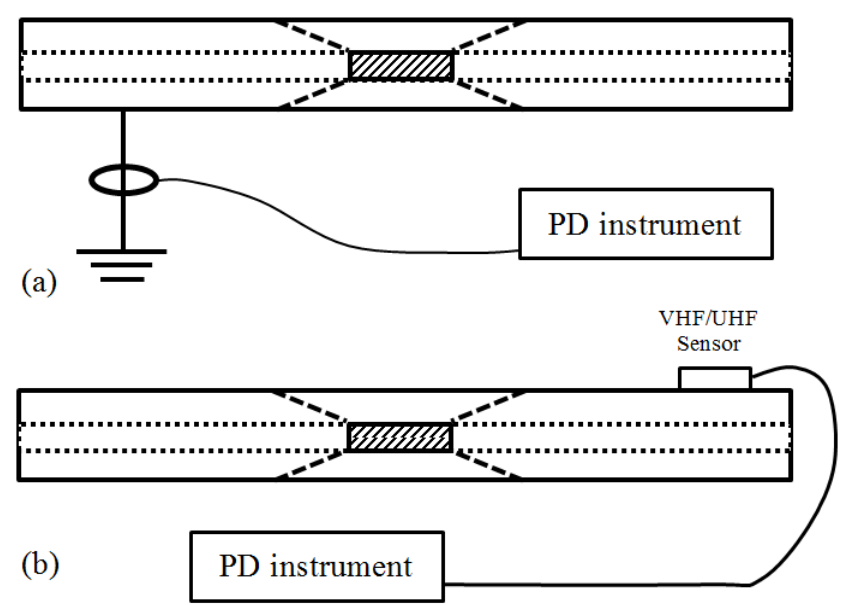

Figure 2. Sketches of PD measurement at a FFJ by means of (a) HFCT applied to a ground connection close to the joint; (b) wireless VHF/UHF sensor without any ground connection close to the joint.

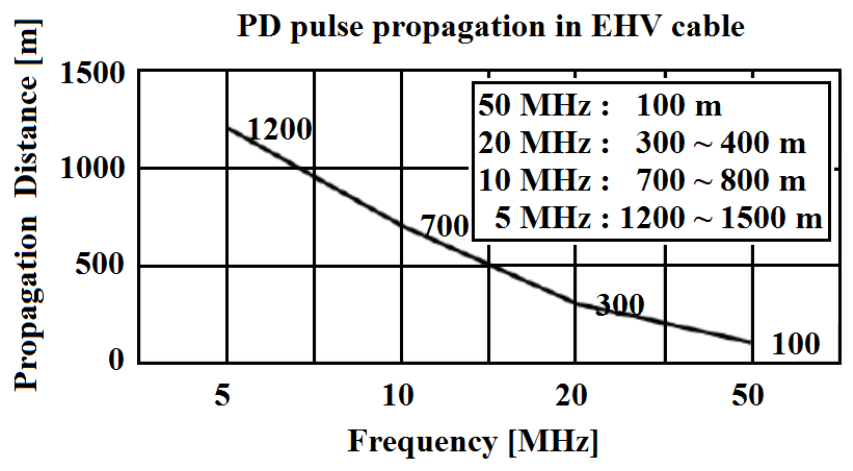

Figure 3. Propagation and attenuation of frequency components of PDs with EHV cable length.

\subsection{PROPOSED TEST PROCEDURE}

The PD measurement shall be made throughout the AC voltage test suggested by CIGRÉ Technical Brochure 496 at $\S$ 5.1 "Routine tests on transmission cables" [5], provided that the insulation system and the cable design allow AC testing. The AC voltage test shall be made at ambient temperature using an alternating test voltage. Since, as stated in Par. 5.1 of [5], "Long manufacturing lengths and high voltage levels may render AC testing impractical", the voltage level, frequency (power or other frequencies) and time of application shall be agreed between the supplier and customer. The test voltage between the conductor and metal screen/sheath shall be raised gradually to the agreed value, which shall then be held for the agreed time.

\subsection{ACCEPTANCE CRITERIA}

At the end of the measurements, a report signed by the manufacturer ${ }^{6}$ giving the test results and including a detailed PD analysis shall be issued. The test shall be successfully passed if the report proves that no critical PD is present in the

\footnotetext{
${ }^{6}$ The manufacturer can agree with the customer that the PD measurement are done by a third party, e.g. an independent test laboratory or a service company. In either case the report shall be under the responsibility and shall be signed by the manufacturer.
}

tested object; by "critical partial discharge" it is meant here a discharge within the insulation of the object under test, excluding all external discharges that can be present during the AC voltage test, e.g. corona discharges due to HV electrodes, conducting leads, etc. related to the test setup.

It must be emphasized that the proposed protocol aims at focusing on the whole PD phenomenon, rather than solely on PD pulse amplitude. Indeed, by stating that the test is passed if the report proves that no critical PD is present, the protocol in fact recommends a thorough analysis of the whole PD phenomenon through the information provided by the wireless instrument (frequency content of PD pulses, pulse shape, phase correlation with applied AC voltage, Phase Resolved PD pattern). This information enables to separate and identify the different PD phenomena possibly detected by the sensor, so as to reject the noise, discard PD phenomena that are not critical for the joint under test (e.g. corona in a nearby termination) and focus on possible critical discharges (i.e. those within the joint insulation). Such critical discharges should be strictly avoided, irrespective of their magnitude.

\section{CONCLUSIONS}

In a continuation of its work on advanced practices for electrical and dielectric diagnostic techniques for testing and characterizing the insulation of polymeric extruded HVDC cables, joints, and terminations, the DEIS Technical Committee "HVDC Cable Systems" has presented:

1) a review of state-of-the-art joint technologies, with analysis of the challenges faced, in the previous Part 1 paper;

2) a novel protocol for PD measurement using AC voltages and $\mathrm{VHF} / \mathrm{UHF}$ electromagnetic sensors for quality control during routine tests on HVDC extruded joints, in the present Part 2 paper.

As far as point 2) is concerned, it should be pointed out that the proposed protocol is a novelty in the technical-scientific literature mainly in the following two respects:

1) it is the first protocol for PD measurement using AC voltages for quality control during routine tests on HVDC extruded joints. In this respect, it fills a gap following the authoritative, but quite generic indications of existing standards on HVDC extruded cable systems;

2) it is the first protocol detailing a PD measurement procedure on cable system components using VHF/UHF EM sensors. In fact, the different polymer dielectric materials typically used in modern joint and termination construction make them specifically amenable for high sensitivity (= void-free) partial discharge measurement using UHF/VHF electromagnetic sensor designs. When two or more overlapping thermoplastic/thermoset polymers are involved in the joint/termination, securely bonded together, void-free interfaces must be assured by PD measurement. For this reason, in the acceptance criteria the protocol states that the test shall be successfully passed if the report proves that no critical partial discharge is present in the tested object. 
It should be also pointed out that excessive details are avoided in the protocol so far, so as to make it as general and flexible as possible. The future experience gained by applying the protocol will enable to refine it and make it more detailed.

It is concluded that the completed review and assessment of a recommended, proposed $\mathrm{VHF} / \mathrm{UHF}$ partial discharge investigation attests to the progress by the Technical Committee in meeting the planned scope of work.

\section{REFERENCES}

[1] G. Mazzanti, G. Chen, J. Fothergill, M. Fu, N. Hozumi, J.H. Lee, J. Li, M. Marzinotto, F. Mauseth, P. Morshuis, I. Troia, A. Tzimas, C. Reed, and $\mathrm{K}$. Wu, "The insulation of HVDC extruded cable system joints. Part 1: review of materials, design and testing procedures," submitted to IEEE Trans. Dielectr. Electr. Insul., Nov. 2018.

[2] G. Mazzanti and M. Marzinotto, Extruded Cables for High Voltage Direct Current Transmission: Advances in Research and Development Power Engineering Series, Wiley-IEEE Press, 2013.

[3] Recommended Practice for Space Charge Measurements in HVDC Extruded Cables for Rated Voltages up to $550 \mathrm{kV}$, IEEE Standard 1732 2017-06-26.

[4] G. Mazzanti, "Space charge measurements in high voltage DC extruded cables in IEEE Standard 1732," IEEE Electr. Insul. Mag., vol. 33, no. 4, pp. 27-33, 2017.

[5] Recommendations for Testing DC Extruded Cable Systems for Power Transmission at a Rated Voltage up to $500 \mathrm{kV}$, CIGRÉ Technical Brochure 496, 2012-04-01.

[6] High Voltage Direct Current (HVDC) Power Transmission Cables with Extruded Insulation and Their Accessories for Rated Voltages up to 320 $\mathrm{kV}$ for Land Applications - Test Methods and Requirements, IEC Standard 62895, 2017-05-11.

[7] X. Gu, S. He, Y. Xu, Y. Yan, S. Hou, and M. Fu, "Partial discharge detection on $320 \mathrm{kV}$ VSC-HVDC XLPE cable with artificial defects under DC voltage," IEEE Trans. Dielectr. Electr. Insul., vol. 25, no. 3, pp. 939-946 2018

[8] IEEE Guide for Partial Discharge Testing of Shielded Power Cable Systems in a Field Environment, IEEE Standard 400.3, 2006-02-05.

[9] Power Cables with Extruded Insulation and Their Accessories for Rated Voltages above $30 \mathrm{kV}\left(U_{\mathrm{m}}=36 \mathrm{kV}\right)$ up to $150 \mathrm{kV}\left(U_{\mathrm{m}}=170 \mathrm{kV}\right)-$ Test Methods and Requirements, IEC Standard 60840, 2011-11-23.

[10]Power Cables with Extruded Insulation and Their Accessories for Rated Voltages above $150 \mathrm{kV}\left(U_{\mathrm{m}}=170 \mathrm{kV}\right)$ up to $500 \mathrm{kV}\left(U_{\mathrm{m}}=550 \mathrm{kV}\right)-$ Test Methods and Requirements, IEC Standard 62067, 2011-11-24.

[11]Electrical Test Methods for Electric Cables Part 3: Test Methods for Partial Discharge Measurements on Lengths of Extruded Power Cable, IEC Standard 60885-3, 2015-04-09.

[12]P. Coors and S. Schierig, "HV AC testing of super-long cables," in Conf. Rec. 2008 IEEE Int. Symp. Electr. Insul. (ISEI), 2008, pp. 636-640.

[13]P. H. F. Morshuis and J. J. Smit, "Partial discharges at DC voltage: their mechanism, detection and analysis," IEEE Trans. Dielectr. Electr. Insul., vol. 12, no. 2, pp. 328-340, 2005.

[14]Bushings for DC Application, IEC/IEEE Standard 65700, 2014-03-19.

[15]High Voltage Test Techniques - Partial Discharge Measurements, IEC Standard 60270-2000, 2000-12-21.

[16]W. Hauschild and E. Lemke, High-Voltage Test and Measuring Techniques, Springer-Verlag, 2014.

[17]M.D. Judd, B.F. Hampton, and O. Farish: "The excitation of UHF signals by partial discharges in GIS," IEEE Trans. Dielectr. Electr. Insul., vol. 3, no. 2, pp.213-228, 1996.

[18]A. Madonia, I. Troia, S. Giannini, E. Riva Sanseverino, S. Franchi Bononi, and G. Mazzanti, "Critical issues in the PD testing methodology for XLPE-insulated MV cables: an experimental case," in IEEE Annu. Rep. Conf. Electr. Insul. Dielect. Phenom. (CEIDP), 2017, pp. 311-314.

[19]A. Madonia, E. Riva Sanseverino, P. Romano, I. Troia, S. Franchi Bononi, M. Albertini, S. Giannini, and G. Mazzanti, "Wireless partial discharge tracking on cross linked polyethylene MV and HV cables," IEEE Electr. Insul. Mag., vol. 34, no. 6, pp. 8-17, 2018.

[20]R. Candela, A. Di Stefano, G. Fiscelli, and G.C. Giaconia, "Portable partial discharge detection device," Patent EP2297589/ WO2009150627, Jun. 11, 2009.
[21]G. Ala, R. Candela, and F. Viola, "Detection of radiated EM transients by exploiting compact spherical antenna features," Recent Patents on Electrical Engineering, vol. 4, pp. 202-208, 2011.

[22]R. Candela, and A.Contin, "A portable instrument for the location and identification of defects generating PD," in Proc. IEEE Electr. Insul. Conf., 2011, pp. 469-473.

[23]HVPD PDs Insight ${ }^{\mathrm{TM}}$ on-line partial discharge handheld test unit, Available at: https://www.cetm.com.my/product/hvpd-pds-insight-online-partial-discharge-handheld-test-unit/

[24] Megger UHD PDD UHF Partial Discharge Detector, Available at: https://www.cetm.com.my/product/megger-uhd-pdd-uhf-partialdischarge-detector/

[25] UltraTEV Partial Discharge Detector, Available https://www.powerpoint-engineering.com/product/ultratev/

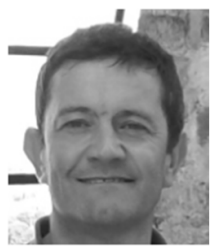

Giovanni Mazzanti (M'04, SM'15) is an Associate Professor of HV Engineering and Power Quality at the University of Bologna, Italy. His interests are life modeling, reliability and diagnostics of $\mathrm{HV}$ insulation, power quality, renewables and human exposure to electro-magnetic fields. Since 2009 he is consultant of TERNA (the Italian TSO) in the HVDC and HVAC cable systems area. He is author or coauthor of more than 200 published papers, and coauthor of the book HVDC Extruded Cable Systems: Advances in Research and Development, Wiley-IEEE Press July 2013. He is chairman of the IEEE DEIS TC "HVDC Cable Systems".

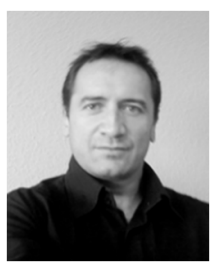

Jerome Castellon (M'08) received his $\mathrm{PhD}$ degree in electronic, optronic and systems from the University of Montpellier (UM), France, in 1997. He has been the technical manager of advanced metrology for electrical engineering "Am2e" company to 2003. Since then, he has been an Associate Professor at UM. Currently, has been working with the Energy and Materials Group "GEM" of the Institute of Electronics and Systems "IES". He has been involved in various research fields related to insulating materials for electrical engineering. His major research activities involve electrical characterization, dielectric phenomena and diagnostic technique developments. He is a member of the IEEE Dielectrics and Electrical Insulation Society, CIGRE SC-D1 and SC-B1, of the French Society of Electrostatics and of the Société des Electriciens et Electroniciens.

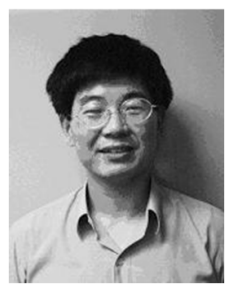

George Chen (SM'11) was born in China in 1961. He received the B.Eng. (1983) and M.Sc. (1986) degrees in electrical engineering from Xi'an Jiaotong University, China. After he obtained the Ph.D. degree (1990) from the University of Strathclyde, UK, on the work of permanent changes in electrical properties of irradiated low-density polyethylene, he joined the University of Southampton as postdoctoral research fellow and became a senior research fellow subsequently. In 1997 he was appointed as a research lecturer and promoted to a Reader in 2002 . He is now the professor of high voltage engineering at the University of Southampton and a visiting professor of Xi'an Jiaotong University. Over the years, he has developed a wide range of interests in high voltage engineering and electrical properties of materials and published over 300 papers. He is active in the IEEE DEIS TC "HVDC Cable Systems".

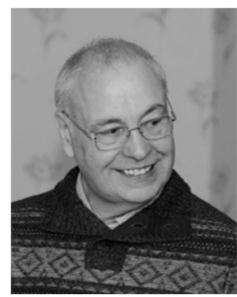

John C. Fothergill (SM'95-F'04) was born in Malta in 1953. He graduated from the University of Wales, Bangor, in 1975 with a Batchelor's degree in electronics. He continued at the same institution, working with Pethig and Lewis, gaining a Master's degree in Electrical Materials and Devices in 1976 and doctorate in the Electronic Properties of Biopolymers in 1979. Following this he worked as a senior research engineer leading research in electrical power cables at STL, Harlow, UK. In 1984 he moved to the University of Leicester where he later gained a personal chair in Engineering In 2012 he moved to City University London where he is Pro Vice-Chancellor (Research and Enterprise). He is active in the IEEE DEIS TC "HVDC Cable Systems". 


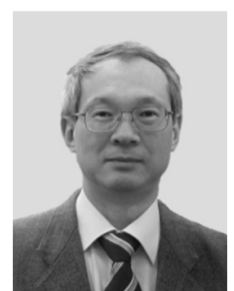

Naohiro Hozumi (M’90) Naohiro Hozumi was born in Kyoto, Japan on 2 April 1957. He received his B.S., M.S. and Ph.D. degrees in 1981, 1983 and 1990 from Waseda University. He was engaged in Central Research Institute of Electric Power Industry (CRIEPI) from 1983 to 1999 . He was an associate professor of Toyohashi University of Technology from 1999 to 2006, and a professor of Aichi Institute of Technology from 2006 to 2011. Since 2011, he has been a professor of Toyohashi University of Technology. He has been engaged in the research in insulating materials and diagnosis for high voltage equipment, acoustic measurement for biological and medical applications, etc. He was awarded in 1990 and 1999 from IEE of Japan for his outstanding research papers. He is a member of IEEE, IEE of Japan and the Acoustic Society of Japan. He is active in the IEEE DEIS TC "HVDC Cable Systems".

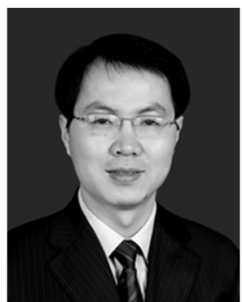

Jian Li (M'05-SM'11) received the M.S. and Ph.D. degrees in electrical engineering in 1997 and 2001, respectively from Chongqing University, Chongqing, China. He is currently a professor and the head of High Voltage and Insulation Technology Department at Chongqing University. His major research interests include online detection of insulation condition in electrical devices, partial discharges, and insulation fault diagnosis for high voltage equipment, environment-friendly liquid dielectrics, anti-icing coating of insulator and transmission line, and polyethylene/organic-montmorillonite nano-composites. He is an author and coauthor of more than 50 journal papers and 50 papers published in proceedings of international conferences. He is active in the IEEE DEIS TC "HVDC Cable Systems".

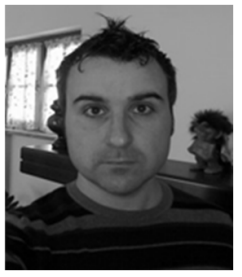

Massimo Marzinotto (S'97, M'01, SM'09) received the Master degree and the Ph.D. degree in electrical engineering at "La Sapienza" University of Roma in 2000 and 2006, respectively. From 2001 to 2008 he joined the Electrical Engineering Department - "La Sapienza" University of Roma. Since 2008 he joined TERNA (the Italian TSO). His main interests are high polymeric materials, power cables, insulators, applied statistics, insulation coordination and transients. He is member of IEEE-DEIS, IEEE-PES, CIGRE, CEI (Italian Electrotechnical Committee) and he is active in different IEEE Committees and CIGRE WGs. $\mathrm{He}$ is author and co-author of different international publications on IEEE transactions and conferences, and coauthor of the book HVDC Extruded Cable Systems: Advances in Research and Development, Wiley-IEEE Press, 2013. He is active in the IEEE DEIS TC "HVDC Cable Systems" and "Outdoor Insulation".

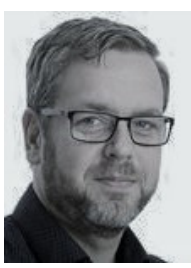

Frank Mauseth (M'11) received his M.Sc. degree in electrical engineering from Delft University of Technology, The Netherlands, in 2001. Since then he has been with the Norwegian University of Science and Technology (NTNU) in Trondheim, Norway, where he also received his Ph.D. degree in 2007 and now is working as an Associate Professor at NTNU. Main fields of interest are high voltage insulation materials and systems, measurement methods and testing. He is active in the IEEE DEIS TC "HVDC Cable Systems".

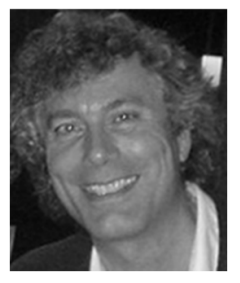

Peter Morshuis (M'95) received the Master degree in electrical engineering from Delft University of Technology. Since 1988, he has been a staff member of the High Voltage Group at Delft University of Technology where he was awarded the Ph.D. degree in electrical engineering in 1993. In 1998, he was a visiting Professor at the University of Bologna. Since 1999, he is an Associate Professor in High Voltage Engineering at Delft University of Technology and he is involved in teaching M.Sc. students in the field of high voltage. In 2012, he started Solid Dielectric Solutions, a company focusing on training/education and consultancy in the field of dielectrics and electrical insulation. His most important fields of interest are HVDC (materials and systems), space charge, partial discharge, aging of electrical insulation and on-line monitoring of high voltage equipment. He is involved in a number of CIGRÉ and IEEE activities. He is an Associate Editor of the IEEE Transactions on Dielectrics and Electrical Insulation and he is active in the IEEE DEIS TC "HVDC Cable Systems".

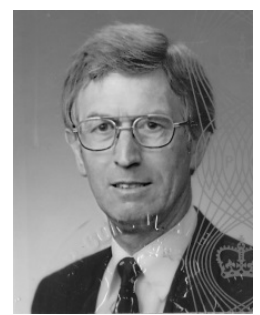

Clive W Reed (M'80) graduated from the University of Bristol, England, in 1956 with a first class special honors BSc degree in chemistry and with a Ph.D. degree in physical chemistry in 1960 . After a post-doctoral fellowship in low temperature physics at the National Research Council of Canada in Ottawa, in 1963 he joined GE Corporate R\&D, Schenectady, NY where he worked on $\mathrm{HV}$ insulation materials and design for numerous commercial applications until retirement in 2002. He is past chairman of the NAS/NRC Washington DC Conference on Electrical Insulation and Dielectric Phenomena, the NAS/NRC Committee on Dielectrics, and CIGRE's Study Committee on Materials for Electrotechnology. His awards include the 2011 Philip Sporn Award "for Cumulative Career Contributions to the Advancement of System Integration in the Theory, Design, and Operation of Large High voltage Electrical Systems in the United States". Presently, he is an independent consultant on HV insulation materials and design. He is active in the IEEE DEIS TC "HVDC Cable Systems".

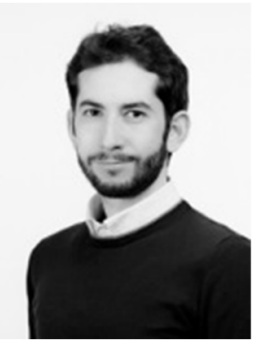

Ivan Troia was born in 1987 in Italy. In 2010 he got the Bachelor degree in Automation Engineering and in 2013 his Master degree in Electrical Engineering from the University of Palermo. Afterwards he joined ABB for few months, he was in the switches and circuit breakers business unit, in the quality department. In July 2014 he joined Prysmian, first as researcher for materials used for HV and telecom application. He was then moved in 2015 in the electrical lab where he was in charge of the MV electrical tests and R\&D tests. From 2016 he is in charge of the HV electrical tests in Milan R\&D. He has cooperations with the University of Bologna and University of Palermo in different research projects and his research topics include partial discharge in AC and DC voltage, space charge measurement, new materials developements and HV test and measuring techniques. He is active in the IEEE DEIS TC "HVDC Cable Systems".

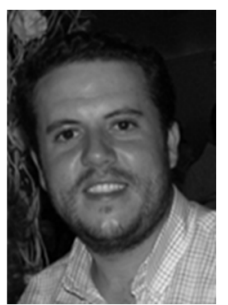

Antonios Tzimas (M'08) received the B.Eng. (2001) and M.Sc. (2003) degrees in electrical and electronic engineering from the University of Leicester where after completing his national service duties in Greece he returned to study for the Ph.D. degree on ageing properties of cross linked polyethylene. He received the degree in 2008. He then joined the National Grid High Voltage laboratory at the University of Manchester as Post-Doctoral researcher till 2013 working on the ageing of outdoor and cable insulation. Currently he is working at Alstom Grid in Materials and HV Technology Research center. In 2011 he became the chair of IEEE Dielectrics and Electrical Insulation Society GOLD membership and part of the society's membership committee. $\mathrm{He}$ is also board member of an annual Conference on Electrical Insulation and Dielectric Phenomena (CEIDP). He is active in the IEEE DEIS TC "HVDC Cable Systems".

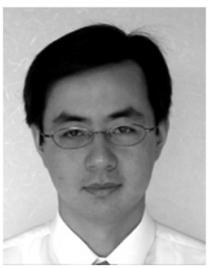

Kai Wu (M'99-SM'13) received the M.S. and Ph.D. degrees in electrical engineering from $\mathrm{Xi}^{\prime}$ an Jiaotong University, China in 1992 and 1998, respectively. He was a postdoctoral fellow from 1998 to 2000 and then joined the staff from 2000 to 2003 at Nagoya University, Japan. In 2003, he worked as a research associate at the University of Leicester, UK. In 2004 and 2005, he was a visiting researcher at the Central Research Institute of Electric Power Industry, Japan. Since 2006, he has become a Professor of Xi' an Jiaotong University, China $\mathrm{He}$ is active in the IEEE DEIS TC "HVDC Cable Systems". 